\title{
The predicted shapes of voids and Xe bubbles in $\mathrm{UO}_{2}$
}

\author{
C. O. T. Galvina, , M. J. D. Rushton ${ }^{\text {b }, ~ M . ~ W . ~ D . ~ C o o p e r ~}{ }^{\text {c }}$, D. A. Andersson ${ }^{\text {c }}$, P. A. Burr ${ }^{\text {d }}$, R. W. \\ Grimes $^{\mathrm{a}}$ \\ ${ }^{a}$ Department of Materials, Imperial College London, London, SW7 2AZ, UK \\ ${ }^{b}$ Nuclear Futures Institute, Bangor University, Bangor, LL57 2DG, UK \\ ${ }^{c}$ Materials Science and Technology Division, Los Alamos National Laboratory, P.O. Box 1663, Los Alamos, NM, 87545, USA \\ ${ }^{d}$ School of Mechanical and Manufacturing Engineering, University of New South Wales, Kensington, 2052, NSW, Australia
}

\begin{abstract}
Morphology is a fundamental attribute when investigating voids and bubbles in $\mathrm{UO}_{2}$. This study uses molecular dynamics and Monte Carlo simulations to predict the lowest energy shapes for voids and bubbles in $\mathrm{UO}_{2}$. The energies of the $\{100\},\{110\}$ and $\{111\}$ surfaces have been calculated and used to predict the equilibrium void shape from Wulff construction. This equilibrium shape is compared to low energy faceted voids exhibiting different relative proportions of each family of terminating surfaces. It is found that the equilibrium Wulff shape does not represent the lowest energy morphology for nm void sizes at temperatures between $300 \mathrm{~K}$ and $1200 \mathrm{~K}$. Furthermore, the lowest energy faceted voids are slightly more energetically favourable than spherical voids, and as Xe is added, and bubble pressure increases, the faceted morphology becomes even more favourable than the spherical shape.
\end{abstract}

\section{Introduction}

Fission and decay products are a consequence of the nuclear chain reaction and a significant proportion of these are accommodated in some way within nuclear fuel. Amongst these are the noble gases $\mathrm{Xe}$ and $\mathrm{Kr}$, which are both insoluble in the $\mathrm{UO}_{2}$ lattice [1-3] leading to a strong thermodynamic driving force to form and grow intra-granular bubbles [4]. Irradiation driven resolution from the bubbles sets up a transfer from bubbles back into the lattice that leads to a flux of gas atoms to the grain boundaries, causing inter-granular bubble formation. These intergranular bubbles grow, interconnect and lead to the release of gas from the $\mathrm{UO}_{2}$ fuel pellet [5]. Important factors include the stability of bubbles and their efficacy as gas atom traps both of which will be influenced by bubble shape.

Intra-granular and inter-granular fission gas bubble formation are performance limiting processes since they degrade the mechanical properties of $\mathrm{UO}_{2}[6,7]$ leading to fuel swelling [6]. There is also a concern that the expansion of fission gas bubbles plays a part in fuel fragmentation and pulverisation during a loss of coolant accident $[10,11]$. Furthermore, fission gas release from the fuel increases the plenum pressure and degrades the thermal conductivity of the fuel-clad gap $[5,6,8,9]$. These effects are likely to become more prevalent as high burnups (> $60 \mathrm{GWd} / \mathrm{t}$ ) become more commonplace.

Email address: (conor.galvin13@imperial.ac.uk) 
A significant portion of the fuel's fission gas inventory is accommodated in bubbles with the remainder dispersed in the $\mathrm{UO}_{2}$ lattice or in the rod free volume. As a result, to quantify the entire fission gas inventory it is important to know the precise amount of gas in bubbles. This becomes even more important during accidents when fuel melting could lead to sudden gas release. Because they are chemically inert, gases are more likely to be released to the environment than other species that are sequestered in a more stable form in the fuel. Moreover, there are significant gaps in our knowledge of the properties and behaviour of fission gas bubbles.

Lab grown single crystals of $\mathrm{UO}_{2}$ exhibit faceted shapes [12] as a consequence of the low energy surfaces present in the crystal. Here we understand the surface energy to be the excess free energy per unit area owing to the existence of a surface at equilibrium. The polar plot of the surface energy as a function of surface normal is known as the $\gamma$ plot. The thermodynamic equilibrium shape of the crystal, also called the Wulff shape or crystal habit, is determined through the Wulff construction from the $\gamma$ plot. This is based on the concept that the crystal seeks to minimise its total surface energy subject to the constraint of fixed volume. A Wulff construction is illustrated in figure 1, where plane normals have been constructed at the ends of the radius vectors corresponding to low energy planes. These planes are known as Wulff planes and correspond to the particular crystal face. The equilibrium shape of the crystal is given by the inner convex hull of all the possible Wulff planes. The distance of each face from the center is thus proportional to the surface energy of that face [13]. Therefore, when the total surface free energy for an isolated particle of a fixed volume in a homogeneous matrix is minimised, the equilibrium shape is determined by the Wulff construction. Following this the question then arises; if the crystals are faceted, why not the voids?

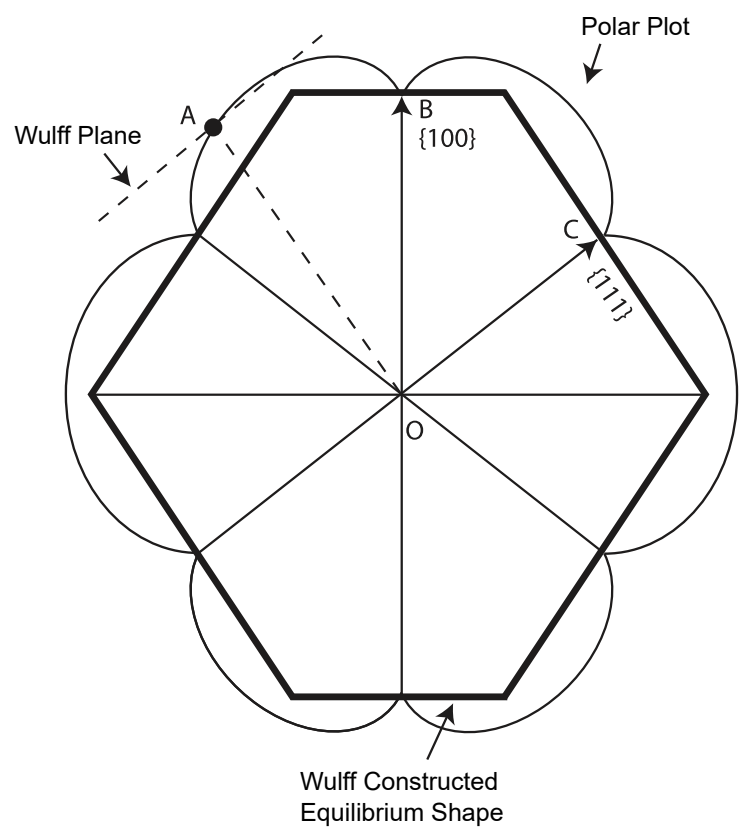

Figure 1: Schematic of Wulff construction in two-dimensions. The length of OA represents a surface energy where the surface normal is proportional to it. Therefore OB and OC represent the surface energies of $\{100\}$ and $\{111\}$, respectively. The Wulff planes are normal to the respective surface energies where the inner envelope of all the Wulff planes give the equilibrium shape. 
Actually, some faceted voids are observed in $\mathrm{UO}_{2}$ at the $\mu \mathrm{m}$ length scale [14-17]. Applying the same underlying physics as above, the inverse Wulff construction can derive or predict the shape of finite voids within crystals. In the case of $\mathrm{UO}_{2}$ the surface energies of $\{100\},\{110\}$ and $\{111\}$ surfaces would dominate the negative shape construction. Here, the negative shape refers to the empty space in a crystal in its equilibrium form. However, the assumption that these Wulff voids are the favoured morphology for faceted voids neglects some energy contributions, for example, the edges where two surfaces meet. These energy contributions could be important, especially for small bubbles and at the atomic scale. Therefore, this paper used a molecular dynamics-Monte Carlo method to investigate different possible faceted shapes in order to establish a protocol by which faceted voids can be created. These voids were then compared to the equilibrium Wulff void predicted from surface energy calculations. Following this, the lowest energy voids were filled with Xe to create bubbles, and a comparison was undertaken between the stability of faceted and spherical morphologies.

\section{Method}

\subsection{Empirical potential for Molecular Dynamics simulations}

Molecular dynamics (MD) void and bubble simulations were carried out using LAMMPS [18], while GULP [19] was used to calculate slab surface energies and entropies. The interatomic forces for $\mathrm{UO}_{2}$ were described using the Cooper-Rushton-Grimes potential [20]. For interactions between the fission products and the $\mathrm{UO}_{2}$ lattice, a potential set derived for use with the CRG model was applied [21]. The interactions between fission products were developed previously by Tang and Toennies [22]. Coulombic interactions were calculated using a Wolf summation with an $\alpha$ parameter of $0.240 \AA$ and a $12 \mathrm{eV}$ cut-off. These parameters were chosen from a convergence test and benchmarked against the particle mesh method.

\subsection{Surface energies from slab calculations}

The surface energies used to predict the equilibrium Wulff shape were calculated from vacuum slabs in GULP. Corresponding vibrational entropies were also obtained from these slabs and were used in the free energy calculations presented later.

The surface energies and entropies were calculated for the $\{110\},\{111\}$ and $\{100\}$ surfaces. These can be defined as Type-I, Type-II and Type-III, respectively, using Tasker's classification scheme [23]. A Type-I surface consists of neutral planes each with equal numbers of cations and anions. Type-II consists of charged planes arranged into groups that give surfaces with no dipole moment normal to the surfaces created by cleaving between these groups. A Type-III surface is charged and has a dipole moment normal to the surface. For Type-III surfaces the most favourable surface termination identified by Abramowski et al. [24] was used throughout. The surface energy can be described as the excess free energy per unit area owing to the existence of a surface at equilibrium and is calculated using equation 1.

$$
E_{\text {surf }}=\left(E_{\text {slab }}-E_{\text {bulk }}\right)\left(\frac{1}{2 \mathrm{~A}}\right)
$$

where $E$ is the energy and $2 A$ is the total slab surface area (due to there being two surfaces as a consequence of the slab configuration). Similarly, the vibrational entropies for the surfaces, which arise from the configuration space occupied by vibrating atoms, were calculated using equation 2 . 


$$
S_{\text {surf }}=\left(S_{\text {slab }}-S_{\text {bulk }}\right)\left(\frac{1}{2 \mathrm{~A}}\right)
$$

Where the subscript slab denotes a supercell containing two surfaces of cross-sectional area $A$, and bulk denotes a supercell of the same size, shape and number of atoms but without cleaved surfaces.

The vibrational entropy of each supercell was calculated using

$$
S^{\mathrm{vib}}=-k_{B} \sum_{n=1}^{3 N-3} \ln \left(\frac{h v_{n}}{k_{B} T}\right)+(3 N-3) k_{b}
$$

where $k_{B}$ is the Boltzmann constant, $h$ is Planck's constant, $T$ is the temperature, and $v_{n}$ is an individual phonon frequency. There are $3 N-3$ phonon frequencies at the gamma point of an energy minimized structure. $S_{\text {slab }}$ and $S_{\text {bulk }}$ were then used within equation 2 to determine the $\{100\} A$ and $\{100\} B$ surface entropies. It should be noted that the definition of surface entropy in equation 2 conserves the number of atoms and, as such, the temperature dependence from equation 3 cancels out.

For the $\{100\}$ surface calculation, the standard $\mathrm{UO}_{2}$ unit cell was used as the elementary structure and $E_{\text {bulk }}$ was calculated by energy minimization at constant pressure of an $8 \times 1 \times 1$ supercell. Subsequently, the supercell volume was extended by $20 \AA$ in the $x$ direction without adjusting Cartesian atomic coordinates, such that two $\{100\}$ surfaces were created. For this length of vacuum, surface energies were found to be well converged. Two oxygen atom terminations were considered and are referred to as $\{100\} \mathrm{A}$ and $\{100\} \mathrm{B}$ in line with Abramowski et al. [24]. The surface supercell was then energy minimized at constant volume to ensure the surfaces remained separated in order to obtain $E_{\text {slab. }}$.

Due to the impact of finite size effects on low frequency phonons, the energy minimized supercells used to calculate $E_{\text {slab }}$ and $E_{\text {bulk }}$ were extended five-fold in each direction parallel to the surface ( $\mathrm{y}$ and $\mathrm{z}$ directions). The phonons of the $\{100\} \mathrm{A}$ and $\{100\} \mathrm{B}$ surfaces and perfect supercells were calculated at the $\Gamma$ point.

The same procedure was carried out for the $\{110\}$ and $\{111\}$ surfaces. However, the elementary fluorite cell was re-oriented such that the surface of interest was always aligned perpendicular to the $\mathrm{x}$ direction. A $6 \times 1 \times 1$ extension of the elementary cell was used for the energy calculation of the $\{110\}$ surface, which was then extended four-fold in the $b$ and $c$ lattice vector directions for the entropy calculation. For the $\{111\}$ surface a $3 \times 1 \times 1$ extension was used to calculate the surface energy, and a four-fold extension in the $b$ and $c$ lattice vector directions for the entropy calculation.

\subsection{Void simulations}

Voids were created using two different methods to obtain spherical voids and faceted shapes composed of different convex combinations of $\{111\},\{110\}$ and $\{100\}$ surfaces.

Spherical void configurations were created by overlaying a sphere on a perfect cubic $\mathrm{UO}_{2}$ structure and deleting the atoms inside. Spherical shapes had diameters equal to 5, 10, 15, 20, 25 and 30 unit cell lattice parameters, corresponding to voids of diameter 2.7, 5.5, 8.2, 10.9, 
13.7 and $16.4 \mathrm{~nm}$, at a temperature of $300 \mathrm{~K}$. Simply deleting atoms from a spherical envelope tends to produce a charged system. In order to restore charge neutrality, oxygen atoms were added or removed at random from the oxygen sites on the sphere surface. As this process is likely to produce high energy surface configurations, these under-went an optimisation procedure to obtain a reasonable starting configuration. Monte-Carlo simulations were performed to optimise the arrangement of surface oxygen sites by swapping the position of surface oxygens between occupied and unoccupied sites on a static lattice using the Metropolis-Hastings algorithm [25]. Following this optimisation, the void was relaxed further using energy minimisation and MD (described below).

Faceted voids were created by a similar method. Faceted voids were created by the intersection of three base shapes, as shown in figure 2. By varying the size of the three base shapes, different faceted volumes are created. The size of the shapes were chosen to be commensurate with the interatomic spacing of $\mathrm{UO}_{2}$. Atoms within these shapes were deleted to produce unoptimised faceted voids. However, rules must be applied to obtain sensible terminating surfaces. For the Type-III $\{100\}$ surfaces, half the oxygen atoms are deleted from the surface, according to the low energy configuration established by Abramowski et al. [24]. The Type-II $\{111\}$ surfaces are based on groups of two oxygen layers above and below a layer of uranium atoms. To avoid a surface dipole these neutral three-layer groups must be preserved. The shape is cleaved between the adjacent $\mathrm{O}$ planes, so that this grouping of stoichiometric and neutral layers is preserved. The $\{110\}$ fluorite surface is type I, so does not need any special treatment. Finally, the edges of the faceted shapes are optimised using a similar procedure described above. As the surfaces are already charge neutral, oxygen atoms were only added and removed to the edges. These edges were then Monte Carlo optimised to create the final void structure. Swaps were performed until the algorithm had converged, which was defined as when the energy of the system did not vary more than $0.02 \mathrm{eV}$ over a 50,000 atom swap window. An example of one of these configurations is shown in figure 3, where the oxygen and uranium atoms are represented by blue and green spheres, respectively. The edge oxygens that can be swapped during the Monte-Carlo run with vacant sites are marked using gold and purple spheres, respectively. 

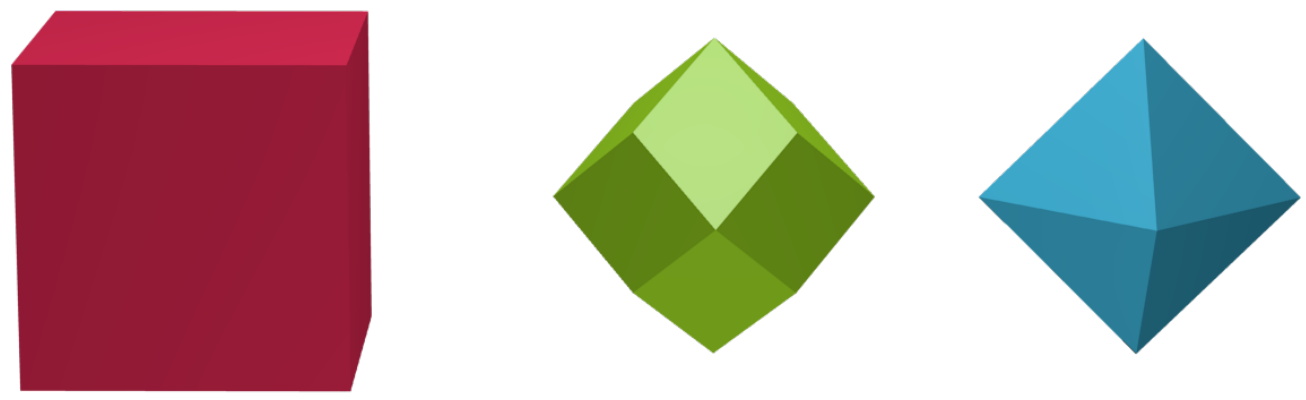

Figure 2: Pure $\{100\},\{110\}$ and $\{111\}$ shapes used for facet construction. 

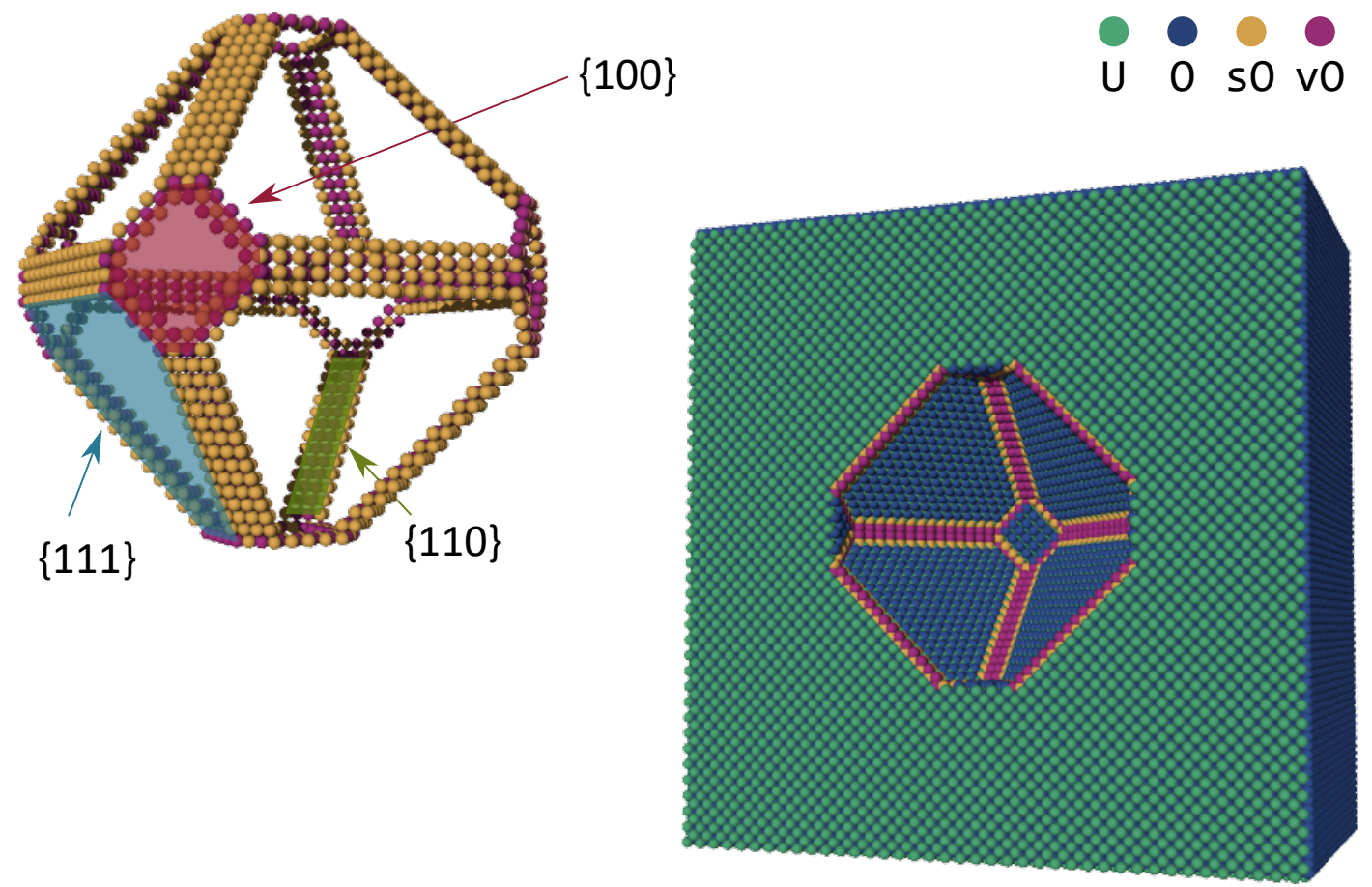

Figure 3: This figure shows a slice through a Wulff void in $\mathrm{UO}_{2}$ and the outer oxygen atoms of the projected Wulff void. Gold atoms are surface oxygens that can be swapped during the Monte-Carlo run with the vacant sites marked in purple. Uranium and oxygen atoms are marked in green and blue, respectively. The image on the left shows the hollow shell of edge atoms without the surrounding bulk. The transparent panes show the different plane types.

To ensure a sufficient sample size was considered to allow reliable inferences to be drawn from the data, a series of faceted voids were generated for three different sizes, a $10 \times 10 \times 10$, a $15 \times 15 \times 15$ and a $20 \times 20 \times 20$ supercell, which defines the basic dimension of the void shapes as described above. Each negative faceted shape was then surrounded by $10 \mathrm{UO}_{2}$ cells in each direction. This allows a brute force approach to search across a large number of shapes and sizes with the intention to capture the energy landscape for a range of shapes beyond that predicted by the Wulff construction. As will be revealed below, this is important as atomic scale effects mean that the lowest energy shape is not governed solely by surface energy concerns.

The resulting supercells containing voids (spheres or faceted structures) were energy minimised at constant volume, then pre-equilibrated at the target temperature for $10 \mathrm{ps}$ using a velocity rescaling algorithm with an initial time-step of $0.001 \mathrm{ps}$, and finally equilibrated for a further 300 ps using a Nosé-Hoover thermostat with relaxation time of 0.1 ps. All equilibrations were carried out at constant volume, with the lattice parameter of the perfect $\mathrm{UO}_{2}$ lattice at the target temperatures ( $300 \mathrm{~K}$ and $1200 \mathrm{~K}$ as predicted from our MD simulations). The total internal system energy (potential energy) was calculated as an average over the last 1 ps of the simulation. 


\subsection{Bubble simulations}

To create bubbles, the voids described above were filled with Xe in randomly generated positions at a density defined as the ratio of the number of Xe atoms to the number of $\mathrm{UO}_{2}$ formula unit vacancies to create the void $\left(\mathrm{Xe}: \mathrm{UO}_{2}\right)$ of $1: 1.25$.

The Xe atoms were added to the void with an initial $3 \AA$ offset between the Xe and lattice atoms (to avoid unphysical interactions due overlap of Xe and lattice ions). A variable timestep NVE simulation was then performed on the Xe atoms in the system (the $\mathrm{U}$ and $\mathrm{O}$ atoms remained static) with a soft-repulsive potential to provide a crude form of energy minimisation to move the Xe atoms apart and further prevent atomic overlap. A cosine pair potential $U\left(r_{i j}\right)$ was chosen as the soft potential for this step and acted between all pairs of Xe ions:

$$
U\left(r_{i j}\right)=A\left[1+\cos \left(\frac{\pi r_{i j}}{r_{c}}\right)\right], r_{i j}<r_{c}
$$

where $r_{i j}$ is the distance between two ions $i$ and $j, A$ establishes the strength of repulsion between the ions and $r_{c}$ is the cut-off. Parameters of $A=1 \mathrm{eV}$ and $r_{c}=1.4 \AA$ were used.

Using a variable time-step the Xe atoms were assigned velocities for a temperature of $10 \mathrm{~K}$, and for the first 5 ps of the MD run, the velocities were scaled at every time-step to maintain this temperature. Then, the temperature was reduced from $10 \mathrm{~K}$ to $0 \mathrm{~K}$ in 5 ps ensuring the gas atoms were static for the final step. The soft cosine potential was then switched off and the Tang-Toennies potential, mentioned in section 2.1, was applied to the Xe atoms.

Again, velocities were assigned for a temperature of $10 \mathrm{~K}$ and an NVE MD run was performed for 500 steps. Keeping the NVE ensemble and using a fixed time-step of 0.002 ps, the Xe atoms were gradually heated to a high temperature of $1500 \mathrm{~K}$ for $4 \mathrm{ps}$, held at this temperature for $4 \mathrm{ps}$ and ramped back down to the target temperature $(300 \mathrm{~K}$ or $1200 \mathrm{~K})$ over a 4 ps period. Initial calculations introduced a repulsive wall between the $\mathrm{UO}_{2}$ lattice and the Xe atoms, described by equation 5 , which was removed once the Xe had equilibrated.

$$
U\left(r_{i j}\right)=\epsilon\left[\frac{2}{15}\left(\frac{\sigma}{r}\right)^{9}-\left(\frac{\sigma}{r}\right)^{3}\right]
$$

where $r$ is the separation between the atom and the region surface, $\sigma$ controls the distance over which boundary-particle repulsion occurs and $\epsilon$ controls the strength of the repulsion. Parameter values of $1 \AA$ and $1 \mathrm{eV} \AA^{-2}$ were use for $\sigma$ and $\epsilon$, respectively. Following this, both the $\mathrm{UO}_{2}$ lattice and the gas were equilibrated for 8 ps at the target temperature.

Once at temperature, NVT dynamics were performed on the whole system for 70 ps using the Nosé-Hoover thermostat (at a value of $0.1 \mathrm{ps)} \mathrm{with} \mathrm{the} \mathrm{total} \mathrm{system} \mathrm{average} \mathrm{potential} \mathrm{energy}$ taken over the last 1 ps. As the system is heterogeneous a global barostat would not describe the system correctly [26]. Thus, an NVT ensemble was used, and care was taken to use the correct lattice parameter for the $\mathrm{UO}_{2}$ matrix surrounding the bubbles at each simulation temperature.

From the equilibrated bubbles structures an $8 \mathrm{~nm}$ diameter spherical bubble was chosen and filled with different $\mathrm{Xe}: \mathrm{UO}_{2}$ ratios (table 1) to understand the radial distribution and the phase of the bubbles by calculating the radial distribution function (RDF). The corresponding hydrostatic bubble pressures, shown in table 1 is one third of the sum over all Xe atoms $i$ of the trace of the atomic stresses: 


$$
P=\sum_{i} \frac{\left(\sigma_{11}^{i}+\sigma_{22}^{i}+\sigma_{33}^{i}\right)}{3}
$$

To calculate the stress term, the LAMMPS compute stress/atom command was used. This produces values from the atomic stress tensors which are multiplied by volume. Thus, to obtain the stress values used in equation 6, they were divided by per-atom volumes. These were calculated as follows: the centre of the bubble was found and the Voronoi tessellation [27] were computed. Using this to identify nearest neighbours, the atoms surrounding the bubble were identified and a polyhedron was calculated from the convex hull of these boundary atoms. The volume of the convex hull's polyhedron was then calculated to give the total volume of the bubble. This was then divided by the number of Xe atoms giving an average atom volume.

Table 1: Ratios and corresponding pressures used for RDF

\begin{tabular}{cc}
\hline Xe: $\mathrm{UO}_{2}$ & Pressure $(\mathrm{GPa})$ \\
\hline 0.1 & 0.006 \\
0.2 & 0.011 \\
0.3 & 0.020 \\
0.4 & 0.059 \\
0.5 & 0.158 \\
0.6 & 0.444 \\
0.7 & 0.937 \\
0.8 & 1.875 \\
0.9 & 3.388 \\
1.0 & 5.537 \\
1.05 & 6.785 \\
1.10 & 7.366 \\
1.15 & 8.316 \\
1.20 & 9.136 \\
1.25 & 9.943
\end{tabular}

\subsection{Energy and entropy calculations}

Both formation and free energies were generated for the analysis of voids and bubbles. The energies were normalised with respect to their corresponding volumes, which can then be reported as energy per $\mathrm{UO}_{2}$ formula unit vacancy. The total formation energy of the void per missing $\mathrm{UO}_{2}$ formula unit $\left(E_{\text {void }}^{\mathrm{f}}\right)$ is given by the following equation:

$$
E_{\text {void }}^{\mathrm{f}}=\left(E_{\text {void }}-\left(\frac{\mathrm{N}_{\text {void }}}{\mathrm{N}_{\text {bulk }}} \cdot E_{\text {bulk }}\right)\right) \cdot\left(\frac{1}{\mathrm{~N}_{\mathrm{V}_{\mathrm{UO}_{2}}}}\right)
$$

where $N_{\text {void }}$ and $N_{\text {bulk }}$ are the number of atoms in the void and bulk structures and $\mathrm{N}_{\mathrm{V}_{\mathrm{UO}_{2}}}$ are the number of $\mathrm{UO}_{2}$ formula unit removed to form the void. $E_{\text {void }}$ and $E_{\text {bulk }}$ are the potential energies of a $\mathrm{UO}_{2}$ supercell containing a void and a bulk $\mathrm{UO}_{2}$ super-cell, respectively.

At finite temperature the free energy governs stability and entropy must be added to equation 7. To include this, the vibrational entropy per unit area for each surface was multiplied by the temperature (i.e. $300 \mathrm{~K}$ or $1200 \mathrm{~K}$ ) and area of corresponding surface, summed, normalised by the number of $\mathrm{UO}_{2}$ formula units needed to make the void and subtracted from the total formation energy calculated in equation 7 . This is represented in equation 8 , 


$$
G_{\text {void }}^{\mathrm{f}}=E_{\mathrm{void}}^{\mathrm{f}}-\left(\left(S_{100}^{\mathrm{vib}} \cdot A_{100}+S_{110}^{\mathrm{vib}} \cdot A_{110}+S_{111}^{\mathrm{vib}} \cdot A_{111}\right) \cdot \frac{T}{\mathrm{~N}_{\mathrm{V}_{\mathrm{UO}_{2}}}}\right)
$$

where $G_{\text {void }}^{\mathrm{f}}$ is the free energy of formation, $S^{\mathrm{vib}}$ is the surface entropy taken from table 2 and $A$ is the area of the surface.

\section{Results}

\subsection{Surface energies}

The equilibrium Wulff shape was predicted using the surface energies. As mentioned earlier, the low energy shape for voids and bubbles should mirror the equilibrium crystal shape. All calculated surface energies and vibrational entropies are presented in table 2.

In this work the CRG potential predicts that for $\mathrm{UO}_{2}$, the $\{111\}$ surface has the lowest surface energy followed by $\{110\}$ and then $\{100\}$. The energy ratio between the $\{100\}$ and the $\{111\}$ surfaces is 1.87 (or 2.1 using the $\{100\}$ B surface). It should be remembered that features such as steps in the surface, not calculated in most surface energy models, may contribute to the surface energy ratio $[16,37]$. Using surface energies from the present calculations reported in table 2, the positive equilibrium Wulff shape (equilibrium shape of a crystal) of $\mathrm{UO}_{2}$ is pure $\{111\}$ (see figure 4 ).

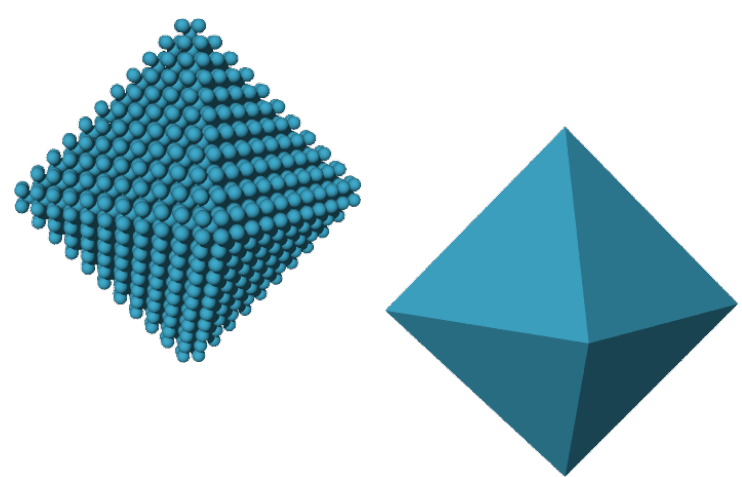

Figure 4: Equilibrium Wulff shape (pure $\{111\}$ ) created using the surface energies from table 2 shown as a configuration made up of atoms and as surfaces.

Using experimental techniques surface energies can be found, however, to the best of our knowledge only studies on polycrystalline $\mathrm{UO}_{2}$ have been carried out and values are reported with large degrees of uncertainty [28]. As it is difficult to accurately measure the energy of each surface plane, measurements for the $\{100\} /\{111\}$ ratio have been reported as: $1.19 \pm 0.01$ [29], $1.27 \pm 0.067$ [30] and $1.42 \pm 0.05$ [16]. Although the ratio differs between different studies, the $\{111\}$ surface is consistently lower energy than the $\{100\}$. This ordering is also supported by observed faceted voids in $\mathrm{UO}_{2}$, which are dominated by the $\{111\}$ surface $[14-17,31,32]$ with smaller expressions of $\{110\}$ and $\{100\}$. 
Table 2: Surface energies $\gamma$ and surface vibrational entropies $S^{v i b}$ from the present study and data from several other MD studies [24, 33-39]. A and B refer to the different terminations of oxygen atoms of the Type-III $\{100\}$ surface [24]. Where previous work report multiple slab sizes, the largest slab size was chosen.

\begin{tabular}{llll}
\hline \multirow{2}{*}{ Surface } & $\begin{array}{l}\left(\mathrm{J} / \mathrm{m}^{2}\right) \\
\gamma \text { Other studies } \\
{[24,33-39]}\end{array}$ & $\begin{array}{l}\left(\mathrm{J} / \mathrm{m}^{2}\right) \\
\gamma \text { CRG }\end{array}$ & $\begin{array}{l}\left(\mathrm{J} / \mathrm{K} / \mathrm{m}^{2} \times 10^{4}\right) \\
S^{v i b} \mathrm{CRG}\end{array}$ \\
\hline$\{111\}$ & $0.86-1.71$ & 1.07 & 1.30 \\
$\{110\}$ & $1.05-2.38$ & 1.65 & 0.95 \\
$\{100\}$ A & $-0.26-2.99$ & 2.00 & 1.54 \\
$\{100\} \mathrm{B}$ & $1.91-3.47$ & 2.25 & 1.91
\end{tabular}

\subsection{Voids and bubbles}

To compare the predicted equilibrium Wulff shape against the faceted voids created by the process outlined in section 2.3, it is important to know the relative energies of voids with different amounts of surface and identify which void configurations are particularly high or low in energy. Ternary plots provide a convenient way to visualise these large amounts of data and are presented for the $20 \times 20 \times 20$ base void dimension in figures 5 and 6 for $E_{\text {void }}^{\mathrm{f}}$ and $G_{\text {void }}^{\mathrm{f}}$ respectively. Energies in these plots are reported per missing $\mathrm{UO}_{2}$ formula unit. For each ternary plot, the lowest energy void structure is marked with a red point. The voids were analysed at $300 \mathrm{~K}$ and $1200 \mathrm{~K}$ (typical centreline fuel pellet temperature) to determine if temperature affected the preferred void morphology.

Figure $5 \mathrm{a}$ shows the potential energy of formation, given by equation 7 , at $300 \mathrm{~K}$. The lowest energy void consists of $63 \%\{111\}$ with the remaining $37 \%$ made up of $\{100\}$ surface. It is worth noting this is different from the equilibrium shape predicted using Wulff construction (marked with a black square), a point that will be discussed later.

As temperature is increased to $1200 \mathrm{~K}$ the energy landscape changes, as shown in figure $5 \mathrm{~b}$. The lowest energy structure at this temperature is made up of $55 \%\{100\}$ surface, $44 \%\{111\}$ surface and $1 \%\{110\}$ surface. Thus, the higher temperature results in an increase in the proportion of $\{100\}$ and $\{110\}$ at the expense of the $\{111\}$.

Figure $6 a$ includes vibrational entropy terms presented in table 2 and therefore, represent the free energy of formation at $300 \mathrm{~K}$ calculated by equation 8 . In figure $6 \mathrm{a}$, the lowest free energy of formation structure (marked with a red point) is the same as the lowest potential energy of formation structure, shown in figure 5a. Furthermore, in figure 6a, the energy difference between the minimum energy structure and the other structures bounded by the $0.18 \mathrm{eV}$ contour is small. The largest energy difference between the minimum energy structure and the other structures in this region is $0.009 \mathrm{eV}$. Those low free formation energy void configurations at $300 \mathrm{~K}$ are made up of $\sim 15 \%-60 \%\{100\}, \sim 40 \%-90 \%\{111\}$ and $<20 \%\{110\}$. Therefore, it is reasonable to say these structures contain mostly $\{111\}$ surfaces followed by $\{100\}$ and then $\{110\}$ terminations (if any).

At $1200 \mathrm{~K}$, the lowest free energy of formation void, shown in figure $6 \mathrm{~b}$, is the same as the lowest potential energy of formation void. The free energy of formation difference between the lowest energy structure and those in the region $0.13 \mathrm{eV}$ is low (up to $0.009 \mathrm{eV}$ ). Thus, as figure $6 \mathrm{~b}$ shows, the preferred amount of surface at $1200 \mathrm{~K}$ is $\sim 50 \%-60 \%\{100\}, \sim 40 \%-50 \%$ 
$\{111\}$ and a small amount of $\{110\}<20 \%$. The lowest energy void structures at $300 \mathrm{~K}$ and 1200 $\mathrm{K}$ are represented in figure 7 . 


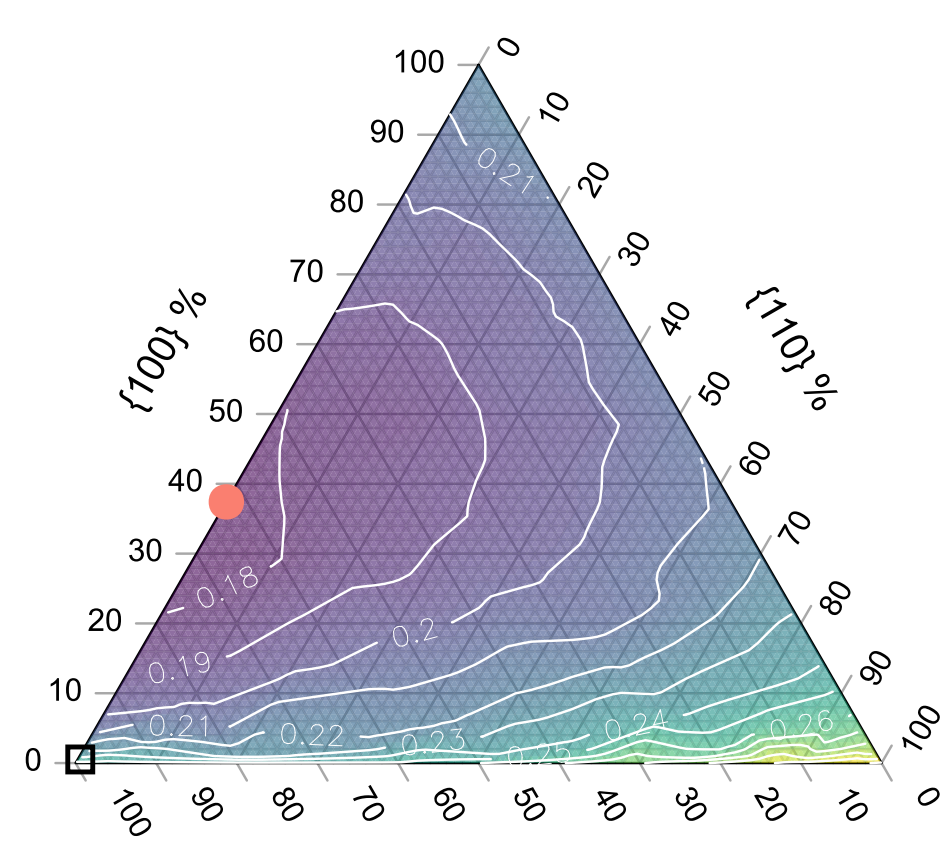

$0.31 \mathrm{eV}$

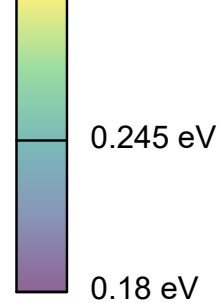

$\{111\} \%$

(a) Potential Formation Energy at $300 \mathrm{~K}$
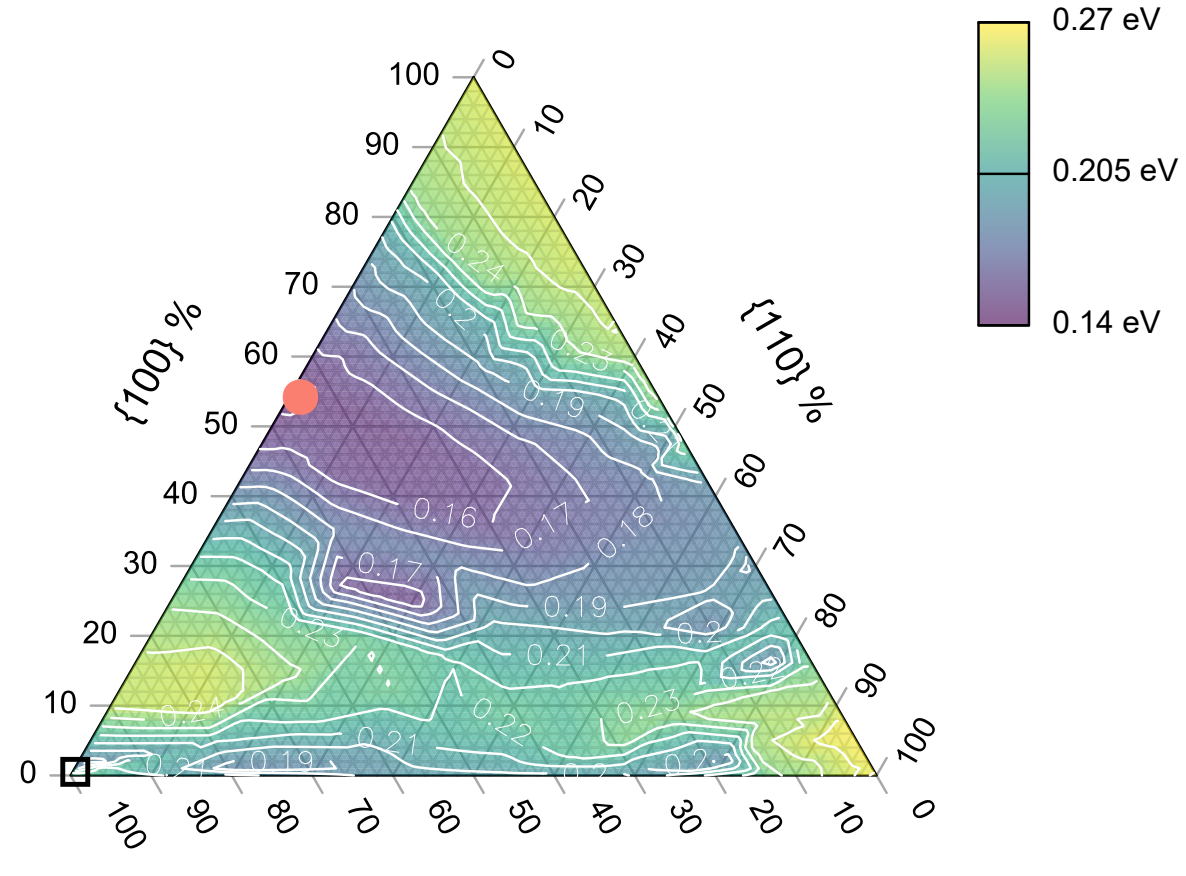

$\{111\} \%$

(b) Potential Formation Energy at $1200 \mathrm{~K}$

Figure 5: $E_{\text {void }}^{\mathrm{f}}$ plotted against the percentage amount of $\{111\},\{110\}$ or $\{100\}$ surfaces at (a) $300 \mathrm{~K}$ and (b) $1200 \mathrm{~K}$. The red circle indicates the lowest energy structure for each ternary plot and the black square indicates the predicted morphology from Wulff construction using the surface energies. 


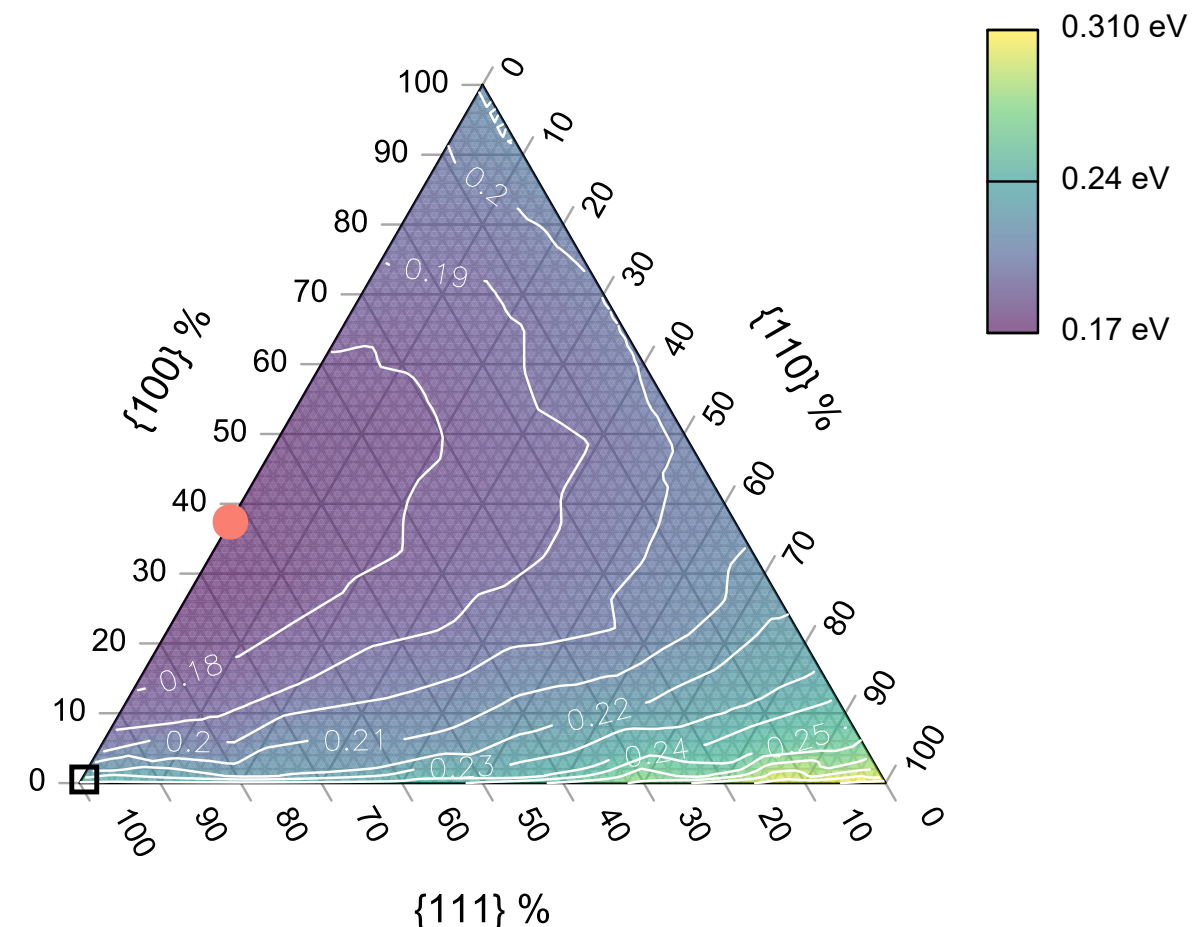

$\{111\} \%$

(a) Free Formation Energy at $300 \mathrm{~K}$

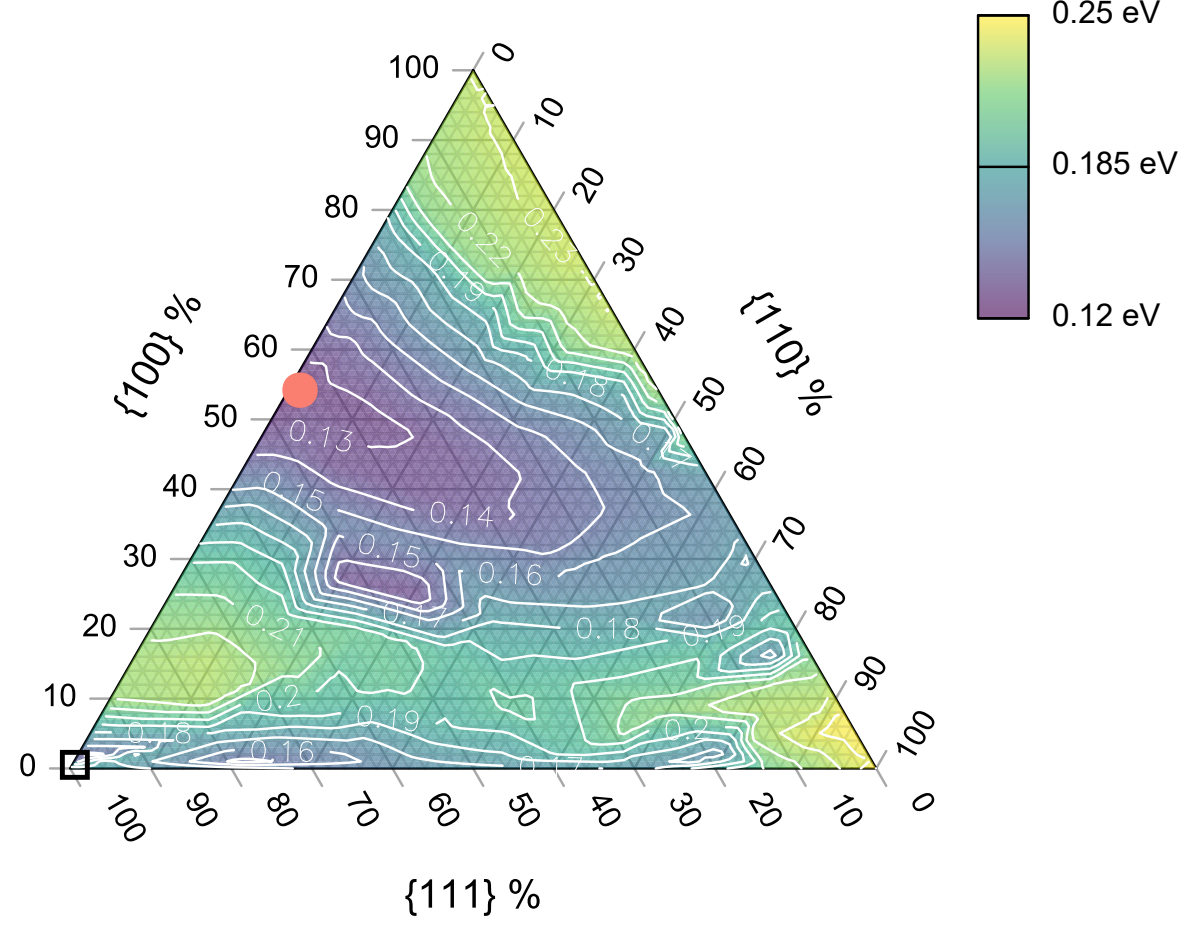

(b) Free Formation Energy at $1200 \mathrm{~K}$

Figure 6: $G_{\text {void }}^{\mathrm{f}}$ plotted against the percentage amount of $\{111\},\{110\}$ or $\{100\}$ surfaces at (a) $300 \mathrm{~K}$ and (b) $1200 \mathrm{~K}$. The red circle indicates the lowest energy structure for each ternary plot and the black square indicates the predicted morphology from Wulff construction using the surface energies. 


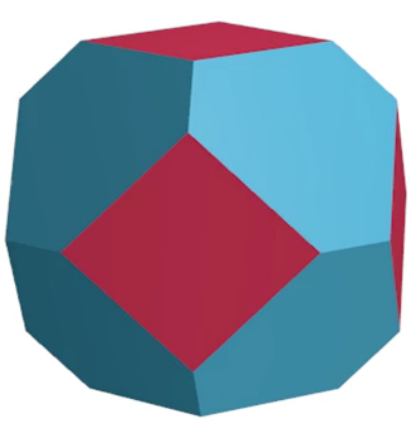

$\mathrm{T}=300 \mathrm{~K}$

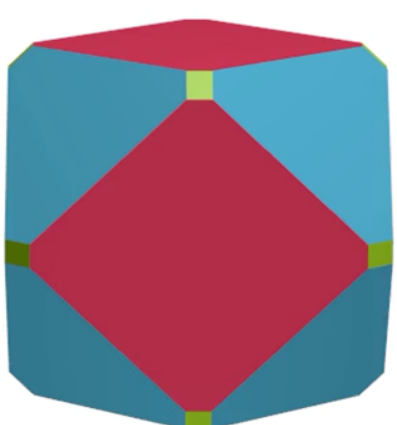

$\mathrm{T}=1200 \mathrm{~K}$

Figure 7: Shape of lowest energy void structures given in the ternary plots. The colours blue, green and red correspond to the $\{111\},\{110\}$ and $\{100\}$ surfaces, respectively.

As the potential energy of formation landscape for the voids in figure 5 is not greatly affected by the vibrational entropy term (shown in figure 6), an assumption is made that only the potential formation energy is required for further analysis. Next, the facet void morphology was compared against spherical void morphology to identify if shape bias exists. This was achieved by plotting the potential formation energy per $\mathrm{UO}_{2}$ formula unit (as was done in the ternary plots) against the number of $\mathrm{UO}_{2}$ formula unit defects needed to create a void. In addition, these voids were filled with Xe atoms to see if bubbles influence shape. As defined by equation 7 , the potential formation energy per missing $\mathrm{UO}_{2}$ formula unit is calculated as the total energy of the configuration containing a void or a bubble minus the energy of an equivalent number of atoms in bulk $\mathrm{UO}_{2}$ (for the bubbles this includes the $\mathrm{Xe}-\mathrm{Xe}$ and $\mathrm{Xe}^{-\mathrm{UO}_{2}}$ lattice interactions).

Figure 8 shows a $\log _{10}-\log _{10}$ plot of the potential energy of formation against the number of $\mathrm{UO}_{2}$ formula unit defects for voids and bubbles of different shapes at $300 \mathrm{~K}$ and $1200 \mathrm{~K}$. For clarity the plot only shows the lowest energy void structures. These were chosen by calculating the convex hull of the potential energy of formation as a function of the number of $\mathrm{UO}_{2}$ formula unit defects for voids from every faceted shape simulated in this study ( 319 structures). For large faceted void configurations (three structures containing $\sim 16,000,26,000$ and $57,000 \mathrm{UO}_{2}$ formula unit vacancies) only the equilibrium Wulff shape was considered, composed entirely of $\{111\}$ surfaces due to performance limitations of running MD simulations for large number of atoms. The main feature of figure 8 is to compare faceted shapes with spheres. Therefore, even if void structures lower in energy than the $\{111\}$ Wulff shape do exist, it does not change the prediction that these voids have a lower energy than the spheres. These low energy voids were then filled with Xe atoms at a Xe: $\mathrm{UO}_{2}$ of 1.25:1 to create the bubbles. 


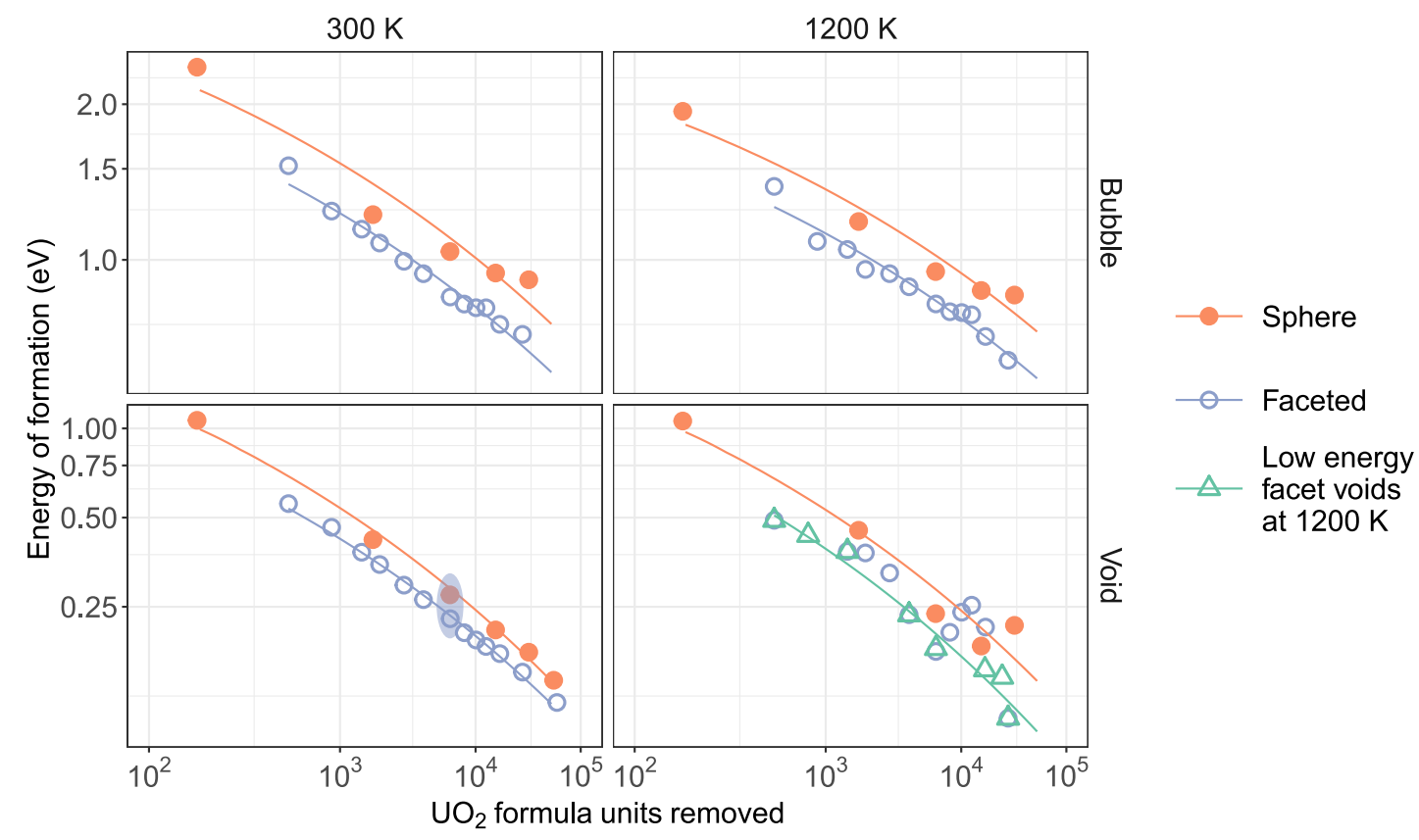

Figure 8: A $\log _{10}-\log _{10}$ plot of the energy (for the bubbles this includes the gas-gas and gas- $\mathrm{UO}_{2}$ interactions) per $\mathrm{UO}_{2}$ formula unit against the number of $\mathrm{UO}_{2}$ formula units to create the void. The lines represent the fit to the data using the functional form in equation 9. The voids enclosed in the transparent purple ellipse used for further analysis in figure 10.

Figure 8 shows that at $300 \mathrm{~K}$ the faceted shape is energetically more favourable than spheres for both voids and bubbles. However, when the lowest energy voids from $300 \mathrm{~K}$ are heated to $1200 \mathrm{~K}$ (represented by purple circles in figure 8), they are no longer the lowest energy structures. That is, the same void configurations are not lowest in energy at both temperatures. This is consistent with the changing energy landscape depicted in figures 5 and 6 . In figure 8 the lowest energy voids at $1200 \mathrm{~K}$ (chosen by taking the structures lying on convex hull described by the $1200 \mathrm{~K}$ data) are shown by green triangles. This indicates that voids would change shape on heating, given enough time, transitioning from their $300 \mathrm{~K}$ shape to their $1200 \mathrm{~K}$ configurations.

The voids represented by the purple circles at $1200 \mathrm{~K}$ were chosen to be filled with Xe to create the bubbles modelled at $1200 \mathrm{~K}$. Even though some spherical voids are energetically lower than these faceted voids (purple circles); once Xe is added this is no longer the case (i.e. the addition of Xe makes sub-optimal faceted bubbles more favourable than spherical bubbles). This suggests that along with the surface energy, the presence of Xe also acts to stabilise the faceted shape, thus these effects are complimentary. Once Xe is added at the pressure considered in this study, faceted bubbles are always favoured. It is important to keep in mind that this is a log plot, therefore, the energy difference between the shapes is larger for a bubble than a void.

The data in figure 8 has been fit with the following power function where the fit coefficients $a_{1}$ and $a_{1}$ and the coefficient of determination, $R^{2}$ are given in table 3 .

$$
f(x)=a_{1} x^{-a_{2}}
$$


Table 3: Fit coefficients $\left(a_{1}, a_{2}\right)$ and a coefficient of determination $\left(R^{2}\right)$ for equation 9 shown in figure 8

\begin{tabular}{cccccc}
\hline Temperature $(\mathrm{K})$ & Type & Shape & $\mathrm{a}_{1}$ & $\mathrm{a}_{2}$ & $\mathrm{R}^{2}$ \\
\hline 300 & Void & Facet & 4.088 & 0.328 & 0.994 \\
300 & Void & Sphere & 5.799 & 0.344 & 0.995 \\
300 & Bubble & Facet & 4.297 & 0.181 & 0.972 \\
300 & Bubble & Sphere & 5.443 & 0.183 & 0.930 \\
1200 & Void & Facet & 4.816 & 0.363 & 0.971 \\
1200 & Void & Sphere & 5.560 & 0.340 & 0.938 \\
1200 & Bubble & Facet & 3.516 & 0.165 & 0.958 \\
1200 & Bubble & Sphere & 4.191 & 0.162 & 0.960
\end{tabular}

An RDF of the Xe atoms for different $\mathrm{Xe}: \mathrm{UO}_{2}$ ratios of an $8 \mathrm{~nm}$ diameter spherical bubble at $300 \mathrm{~K}$ is plotted in figure 9. At the ratio of $1.25: 1$ the Xe atoms exist as a solid as shown by the characteristic peaks associated with a crystalline material. It is important to understand that at high enough pressures the bubble is a highly defective system exhibiting a close-packed structure containing many stacking faults and a number of Xe nano-crystallites. These subtle changes in the structure of the Xe bubbles, as the gas density changes, is apparent in the RDF. A small number of crystallites with low misorientation would give a well-defined RDF. However, the peak heights can reduce depending on the amount and orientation of crystallites. Above a $\mathrm{Xe}: \mathrm{UO}_{2}$ ratio of $0.6: 1$ (this was confirmed by visual characterisation) the bubbles contain varied amount of packed structures consistent with a solid structure. However, the peak height changes in the RDF and may indicate bubble Xe: $\mathrm{UO}_{2}$ ratios that favour order of a Xe lattice (with narrower, taller peaks indicating lower variation between atomic environments and hence greater order). Figure 10 shows a plot of the potential energy of formation difference ${ }^{1}$ between a faceted and spherical bubble of similar volume against $\mathrm{Xe}: \mathrm{UO}_{2}$. The two structures used are highlighted by the purple transparent ellipse in figure 8 . There is a relationship showing that as Xe is added, the energy difference between these two configurations increases. This suggests that faceted shapes should be observed for bubbles that contain solid Xe. That is, the faceted morphology is more favourable than spheres for voids, but the potential energy difference is small. When Xe atoms are added this difference in potential energy between the faceted configuration and the spherical configuration increases suggesting it becomes more likely that the Xe atoms would prefer to occupy a faceted shaped structure. As the pressure decreases in this bubble, the potential formation energy difference decreases. When the pressure decreases further, and there is no solid within the bubble, the energy difference converges to the same value as if the bubble had no Xe (i.e. a void). Figure 11 shows the predicted pressure-Xe:UO $\mathrm{U}_{2}$ ratio relationship for a spherical bubble of $8 \mathrm{~nm}$ diameter. The blue dashed line indicates the boundary where a solid phase is first identifiable in the bubble. This was confirmed visually (using OVITO visualisation software [40]) and by the emergence of a small broad peak in the $\mathrm{RDF}$ centred around an interatomic separation of $\sim 0.55 \mathrm{~nm}$. This corresponds well with the interlayer spacing along $\langle 100\rangle$, meaning the peak position is equal to the lattice parameter of the Xe assuming an FCC lattice. This was calculated as $\frac{4 r}{\sqrt{2}}=0.54 \mathrm{~nm}$, for a Xe atomic radius $r=0.19 \mathrm{~nm}$, obtained from the position of the first peak in the RDF (which can be calculated as $2 r$ ). The solid blue line in figure 11 indicates when the bubble becomes a more ordered solid (characterised by sharper peaks in the RDF). Based on Xe phase diagrams [41, 42] the Xe solid phase at $300 \mathrm{~K}$ is observed at a pressure of $\sim 0.51 \mathrm{GPa}$. This agrees well with our observation

\footnotetext{
${ }^{1}$ The potential energy of formation per $\mathrm{UO}_{2}$ formula unit of the faceted bubble minus that of a spherical bubble.
} 
of solid in the bubble seen above a pressure of $0.44 \mathrm{GPa}$. For pressures considered in this study no dislocation punching was observed, however, a very small number of defects were created in the anion lattice at the interface between the Xe and the $\mathrm{UO}_{2}$ matrix.

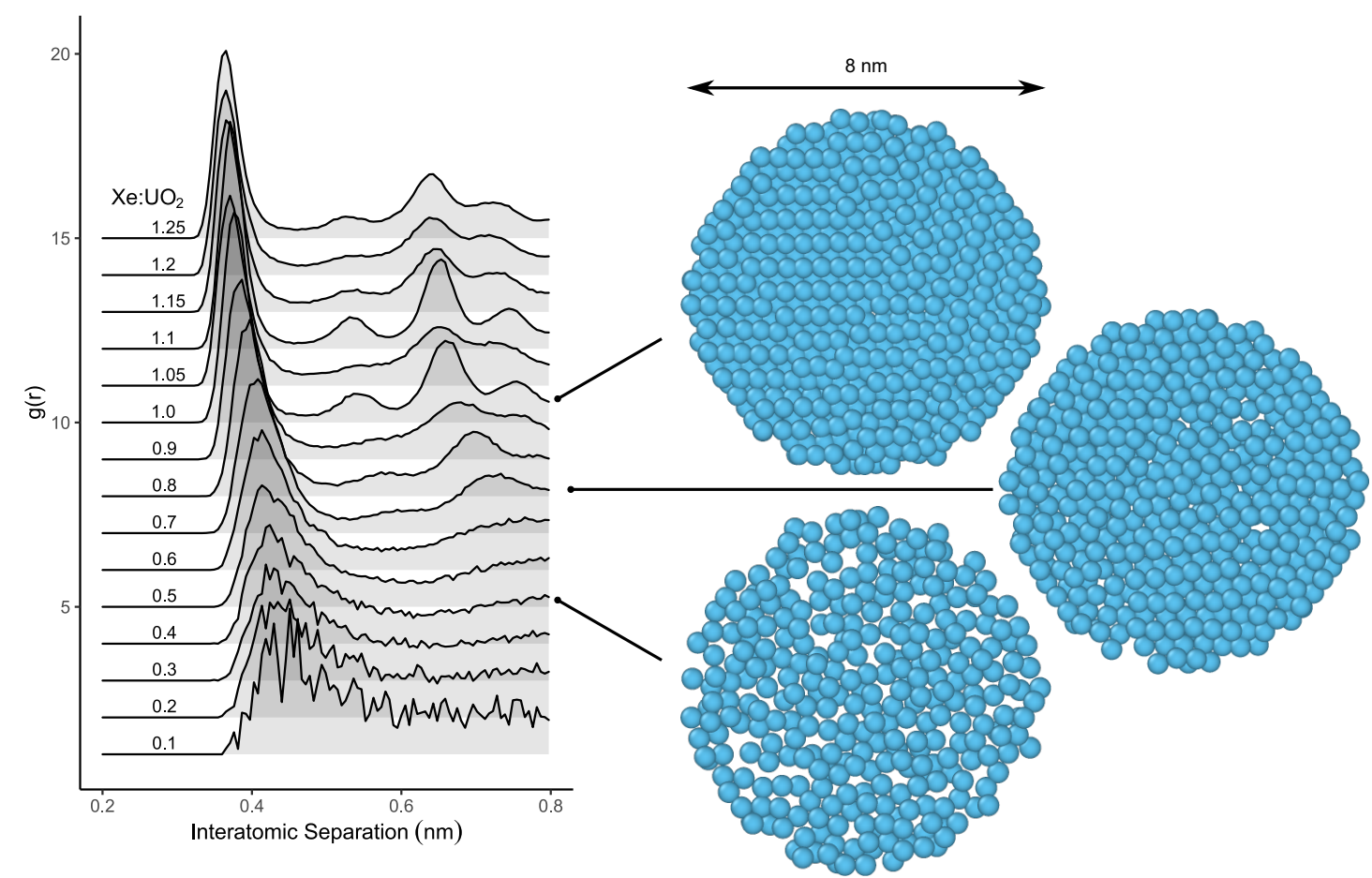

Figure 9: A plot of the Xe RDF for different $\mathrm{Xe}: \mathrm{UO}_{2}$ ratios of spherical bubble.To normalise the RDFs an assumption is made that the volume of a sphere (for the void size) is calculated relative to the cell. Three slices have been taken through the equator of three bubbles in isolation from the $\mathrm{UO}_{2}$ matrix with the Xe atoms represented by blue spheres. Each slice $0.05 \mathrm{~nm}$ thick and viewed along $\langle 110\rangle$. This corresponded with the normal of the most densely packed Xe atomic layers. 


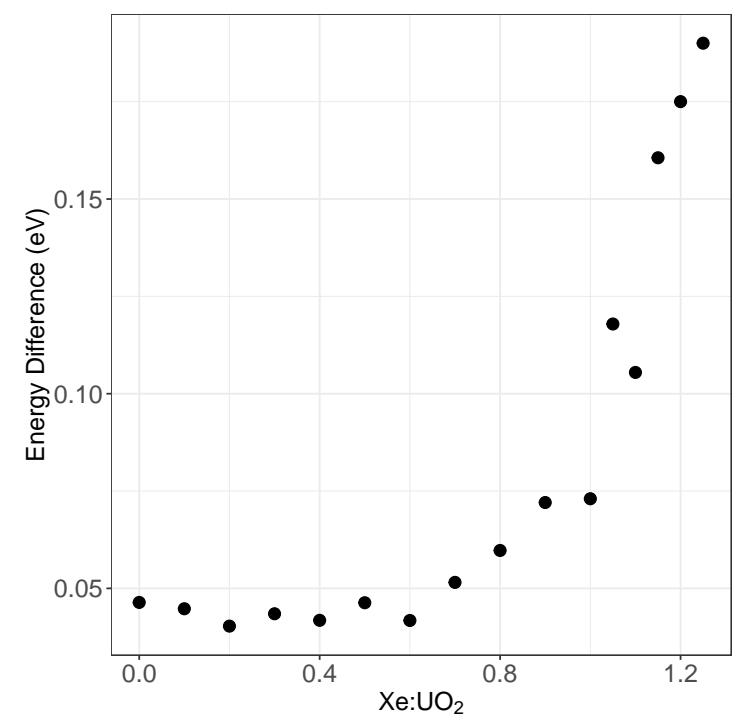

Figure 10: A plot of the energy difference between faceted and spherical bubbles, highlighted in figure 8 , for different $\mathrm{Xe}: \mathrm{UO}_{2}$ at $300 \mathrm{~K}$.

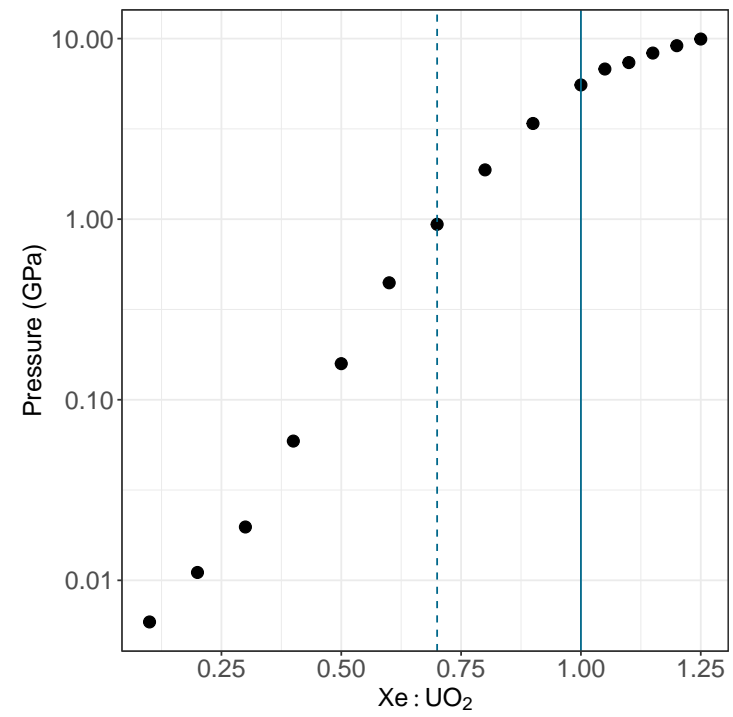

Figure 11: The pressure as a function of bubble $\mathrm{Xe}: \mathrm{UO}_{2}$ ratio. The blue dashed line corresponds to where the bubble starts to become solid and peaks begin to appear in the RDF. The blue solid line represents where a more clearly defined RDF becomes apparent and bubble is a more ordered solid. The y-axis has been scaled by $\log _{10}$. These are the corresponding pressures for each $\mathrm{Xe}: \mathrm{UO}_{2}$ in the RDF.

\section{Discussion}

For the structures considered in this study, there is a clear prediction that voids and bubbles containing Xe favour faceted morphology. Although it has previously been assumed that, based 
solely on surface energy concerns, the equilibrium Wulff shape void would have the lowest energy, this is not supported by the current study. The current results indicate that other energy terms are important. For instance the energy due to atomic arrangement found at the edges where each surface facet meets its neighbours. The Wulff method is based on a continuum description of the material and does not account for discrete atomic effects. For macroscopic crystals it is likely that this edge energy is small in comparison to the surface energy making it reasonable to ignore. At the nanometre length scales considered here, this assumption may break down as energy contributions of edges may be much closer to those of surfaces due to the lower surface areas involved. Therefore, by sampling a large number of faceted voids and optimising the edges using Monte-Carlo swaps this study was able to compare faceted voids, which include this energy term, with the equilibrium Wulff void predicted from surface energy calculations. Nevertheless, at greater sizes there will be other effects such as steps, which are not simulated here $[16,37]$.

The ternary plots in figures 5 and 6 show the energy landscape for simulated faceted voids. There are clearly regions containing void shapes that exhibit lower energy than the predicted equilibrium Wulff shape, which is pure $\{111\}$. Indeed, all of the faceted voids consisting of only one type of surface, $\{111\},\{100\}$ or $\{110\}$, are high energy structures. So the low energy morphologies contain a mixture of these surfaces and are temperature dependent. For example, at $300 \mathrm{~K}$ in figures 5 and 6 , the lowest energy structure was predicted to be a truncated octahedron made up of $63 \%\{111\}$ with the remaining $37 \%$ made up of $\{100\}$ surface. This is consistent with what is seen in experiment [16] and by continuum computer simulation [43]. The lowest energy void at $1200 \mathrm{~K}$, which is more akin to the centreline temperature of a fuel pellet in a reactor, consists of $55 \%\{100\}$ surface, $44 \%\{111\}$ surface and $1 \%\{110\}$ surface.

Comparing faceted voids with spherical voids, as was done in figure 8 , it is predicted that faceted voids are favoured. For large length scales $(\mu \mathrm{m})$ faceted voids are expected at equilibrium as shown by Castell [16], Van Lierde [29] and Padden [14, 15]. Moreover, 'spherical' voids viewed by electron microscopy of fractured surfaces of polycrystalline uranium oxide $[31,32]$ have well developed $\{111\},\{110\}$ and $\{100\}$ planes on their surface [15]. Furthermore, MD predictions by Chartier, Van Brutzel and Freyss [39] found that voids in $\mathrm{UO}_{2}$ with a diameter between $1.2 \mathrm{~nm}$ and $1.5 \mathrm{~nm}$ can be expected to be faceted. Of course, at very small bubble diameters it is only possible to very roughly approximate a sphere due to the need to accommodate the grid like structure of the fluorite lattice; consequently the envelope of small bubbles are necessarily a patchwork of different crystallographic surfaces. The results presented in the current work consider somewhat larger voids and bubbles, from $\sim 3 \mathrm{~nm}$ to $\sim 16 \mathrm{~nm}$, and clearly indicate that a faceted morphology is favoured in this size range for the conditions considered.

When the Xe atoms are added to these voids to produce bubbles, our results continue to predict that faceted shapes are more favourable than their spherical counterparts. Indeed, as figure 10 shows, the energy difference between a spherical bubble and a faceted bubble of a similar size tends to increase as the bubble pressure increases. That is, as more Xe atoms are introduced into the void, the greater the difference in energy between the spherical and faceted shapes. Using the RDF in figure 9 and the corresponding pressure-Xe: $\mathrm{UO}_{2}$ ratio curve in figure 11, a solid phase is identified for an $8 \mathrm{~nm}$ diameter void at $\mathrm{Xe}: \mathrm{UO}_{2}$ ratios greater than $0.6: 1$. Linking this to the energy difference between a spherical bubble and a faceted bubble of similar volume, shown in figure 10, it can be reasoned that when the Xe atoms are not in a solid phase the energy difference is the same as if the bubbles were voids. This is consistent with the smaller interaction expected between gas atoms and the boundary imposed by the $\mathrm{UO}_{2}$ ma- 
trix. When the pressure of the Xe atoms is sufficient and a solid phase appears in the bubble, the energy difference is larger than if the bubble were gaseous or a void. Finally, when most of the Xe atoms are a solid and there are many Xe atoms occupying the available volume and we predict the largest increase in energy difference, as more Xe is added this increase becomes more rapid. This could suggest that when many faceted bubbles are observed experimentally the Xe atoms are at a high pressure (greater than $2.5 \mathrm{GPa}$ ) and are in a solid state. Even though faceted shapes are predicted to be more favourable for voids, it is important to bear in mind that the energy difference between being a sphere or faceted is small. This is also true for when the Xe atoms are a gas. Thus, there could be a variety of shapes observed experimentally due to other local stress factors. For example, local strain effects might tip the balance between different faceted morphologies, and thus across the fuel pellet we should expect variation in shapes with the proportion of different surfaces being slightly or somewhat different. Of course, other influences like local stoichiometry might also influence the balance and could be investigated in future.

As already highlighted, faceted bubbles are seen experimentally in $\mathrm{UO}_{2}$ [44-47]. Miao et al. [44] presented STEM HAADF and TEM BF images that show large $(\sim 16 \mathrm{~nm})$ faceted intragranular Xe bubbles alongside small $(\sim 2 \mathrm{~nm})$ spherical bubbles. These faceted bubbles have a morphology related to the shape predicted in this study (i.e. $\{111\}$ octahedron truncated by $\{100\}$ planes). Similarly, He et al. [45] show $\mathrm{Kr}$ bubbles $(\sim 5 \mathrm{~nm})$ in $\mathrm{UO}_{2}$ that exhibit facets that consist of $\{111\}$ and $\{200\}$ planes. Both these experiments were carried out under ion irradiation. Further, in a paper by Baker [46] small $(\sim 2 \mathrm{~nm})$ intra-granular bubble morphology in $\mathrm{UO}_{2}$ irradiated in a reactor, at temperatures $<2070 \mathrm{~K}$ was octahedral in shape with faces parallel to $\{111\}$ and $\{100\}$. It was assumed that these facets can cause bubble immobility [46] and at annealing temperatures greater than $1970 \mathrm{~K}$, bubbles of diameter 5-20 $\mathrm{nm}$ (where the bubbles are spherical) bubble movement was observed. Therefore, understanding the shape of these bubbles is important. Furthermore, in a paper by Wood [47] for irradiated $(\mathrm{U}, \mathrm{Pu}) \mathrm{O}_{2}$ fuel in the temperature range 1773-2273 K, there was a high concentration of small faceted gas bubbles with little evidence of bubble motion. Further evidence of faceted bubbles was conceptualised by Goodhew [48] who deduced that all bubbles for which the growth is limited by the collection of vacancies, will tend to become faceted onto low energy faces predicted by Wulff construction. That implies that most gas bubbles which are large enough for their shape to be resolved appear to be faceted and spherical shaped bubbles are seen at temperatures where vacancy transport is rapid and kinetic surface effects dominate.

Other MD studies [39, 49] have considered Xe and He faceted and spherical bubbles. One of the key findings in those studies was that spherical bubbles of $\mathrm{Xe}$ in $\mathrm{UO}_{2}$ with a diameter between $1.2 \mathrm{~nm}$ and $1.5 \mathrm{~nm}$ are the most stable shape compared to different morphologies. However, those faceted bubbles were either of pure $\{111\},\{110\}$ or $\{100\}$ surfaces or a Wulff shape constructed from calculated surface energies. The results in this paper show these are not the lowest energy configurations. At this size, atomistic effects matter. Furthermore, the previous MD studies found that He filled bubbles with diameters $<5 \mathrm{~nm}$ favour a truncated octahedron shape (i.e. a shape with $\{111\}$ facets), whereas bubbles greater than this diameter are spherical (a $5 \mathrm{~nm}$ bubble roughly corresponds to a size of $\sim 1778$ removed $\mathrm{UO}_{2}$ formula units as by our classification in figure 8). One essential difference between the previous MD work and the work presented here, other than the different species of gas atom, is that they started from a spherical configuration. The facet shape obtained previously [39, 49] for smaller bubbles formed as a consequence of MD equilibration. It could be the case that as the bubble diameter increases the timescale necessary for the bubble to restructure to a faceted shape has 
become too long. The kinetics of $\mathrm{UO}_{2}$ could be too slow for an $\mathrm{MD}$ simulation to capture the change of morphology from spherical to faceted. Thus, the starting configuration matters.

\section{Conclusions}

Void and bubble morphology is an important characteristic to consider when investigating $\mathrm{UO}_{2}$ fuel performance. This paper outlines a method to create spherical and faceted voids, using Monte-Carlo swaps to sample different combinations of atoms on the surface of spheres and at the edges of faceted voids where surfaces meet to create structures with a low energy. These structures are available at https://www.atomsim.org/bubbles. Potential energies of formation and free energies of formation of all the faceted voids were compared to find preferred shapes. It was found that the lowest energy structure at $300 \mathrm{~K}$ consisted of $63 \%\{111\}$ surface with the remaining $37 \%$ made up of the $\{100\}$ surface: at $1200 \mathrm{~K}$ the lowest energy structure was made up of $55 \%\{100\}, 44 \%\{111\}$ and $1 \%\{110\}$. These structures do not correspond to the equilibrium Wulff shape calculated from surface energies, which in this study consists of only $\{111\}$ facet. Therefore, we predict other energy terms have an influence at the atomic scale, in particular at the edges where surfaces meet.

The total formation energy of all the 319 faceted voids created in this study were compared to spherical shapes: it was found that the faceted voids had a lower energy. However, the potential energy difference between the two shapes was small $(<0.05 \mathrm{eV})$, which is consistent with the experimental observation of both faceted and spherical voids in $\mathrm{UO}_{2}$. Although temperature affects the make-up of the lowest energy faceted void, it does not change the result that faceted voids are lower in energy than spherical voids at $300 \mathrm{~K}$ and $1200 \mathrm{~K}$, the temperatures considered in this study.

For both temperatures, spherical and faceted voids were filled with Xe atoms to create bubbles at a Xe: $\mathrm{UO}_{2}$ ratio of 1.25:1. Again, faceted bubbles provided the lower energy structures. Interestingly, for the bubbles the energy difference between a facet and a sphere was larger than for the voids. To investigate this effect, a spherical void and a faceted void of similar volume were filled with Xe at different $\mathrm{Xe}: \mathrm{UO}_{2}$ ratios. It was found that as more Xe was added, the energy difference between the two shapes increased in favour of the facet. By choosing a bubble morphology (in this case a $8 \mathrm{~nm}$ diameter sphere) and representing the different $\mathrm{Xe}: \mathrm{UO}_{2}$ ratios with an RDF, the behaviour of the Xe atoms was predicted. This revealed that as more Xe was added to the bubble it a solid Xe phase could be identified at Xe: $\mathrm{UO}_{2}$ ratios greater than 0.6:1. Relating this information to the energy difference between the sphere and faceted shapes, it was seen that when the Xe atoms behaved as a gas the energy difference was the same as if they were voids. It is not until the $\mathrm{Xe}: \mathrm{UO}_{2}$ ratio is greater than 0.6:1 that a larger energy difference occurs, and this difference is at its largest when most of the Xe atoms are in the ordered solid state $\left(\mathrm{Xe}: \mathrm{UO}_{2}\right.$ ratio greater than 1.1).

The pressures corresponding to the $\mathrm{Xe}: \mathrm{UO}_{2}$ ratios are shown in the results section. From this pressure values can be applied to what was already observed. For example, when Xe is in the solid state it has a pressure of $\sim 5.5 \mathrm{GPa}$ and over. Thus, a proposition can be made that when many faceted bubbles are observed experimentally at the nanometre scale, the Xe atoms are in a solid state and at a pressure over 5.5 GPa. As well as outlining the shape of a void and bubble with size, this study shows that it is important to include low energy faceted voids 
and bubbles in future simulations ${ }^{2}$. Nevertheless, features such as steps in void and bubble surfaces as well as the interaction between lattice defects and the bubble must be included in future work.

\section{Acknowledgements}

Computational resources were provided be the Imperial College High Performance Computing Service. This work was undertaken with the assistance of resources and services from the National Computational Infrastruc- ture (NCI), which is supported by the Australian Government; the Multi-modal Australian ScienceS Imaging and Visualisation Environment (MASSIVE); the Pawsey Supercomputing Centre, which is supported by the Australian Government and the Government of Western Australia; and was enabled by Intersect Australia Limited. Funding for MWDC and DAA was provided by the US Department of Energy, Office of Nuclear Energy NEAMS (Nuclear Energy Advanced Modeling and Simulation) and CASL (Consortium for Advanced Simulation of Light Water Reactors) programs. Los Alamos National Laboratory, an affirmative action/equal opportunity employer, is operated by Triad National Security, LLC, for the National Nuclear Security Administration of the U.S. Department of Energy under Contract No. 89233218CNA000001. MJDR was funded as part of the Sêr Cymru II programme funded through the Welsh European Funding Office (WEFO) under the European Development Fund (ERDF).

\section{References}

[1] C. R. A. Catlow. Fission Gas Diffusion in Uranium Dioxide. Proceedings of the Royal Society A: Mathematical, Physical and Engineering Sciences, 364(1719):473-497, dec 1978.

[2] R. W. Grimes and C. R. A. Catlow. The Stability of Fission Products in Uranium Dioxide. Philosophical Transactions of the Royal Society A: Mathematical, Physical and Engineering Sciences, 335(1639):609-634, jun 1991.

[3] D. C. Parfitt and R. W. Grimes. Predicted mechanisms for radiation enhanced helium resolution in uranium dioxide. Journal of Nuclear Materials, 381(3):216-222, nov 2008.

[4] S. T. Murphy, A. Chartier, L. Van Brutzel, and J.-P. Crocombette. Free energy of Xe incorporation at point defects and in nanovoids and bubbles in $\mathrm{UO}_{2}$. Physical Review B, 85(14):144102, apr 2012.

[5] J. Rest, M. W. D. Cooper, J. Spino, J. A. Turnbull, P. Van Uffelen, and C. T. Walker. Fission gas release from $\mathrm{UO}_{2}$ nuclear fuel: A review. Journal of Nuclear Materials, 513:310-345, jan 2019.

[6] D. R. Olander. Fundamental aspects of nuclear reactor fuel elements. Technical report, Technical Information Center, U.S. Department of Energy, jan 1976.

[7] V. Rondinella and T. Wiss. The high burn-up structure in nuclear fuel. Materials Today, 13(12):24-32, dec 2010.

\footnotetext{
${ }^{2} \mathrm{~A}$ repository https://www.atomsim.org/bubbles has been created containing faceted void structures
} 
[8] C. Bagger, M. Mogensen, and C. T. Walker. Temperature measurements in high burnup $\mathrm{UO}_{2}$, nuclear fuel: Implications for thermal conductivity, grain growth and gas release. Journal of Nuclear Materials, 211:11-29, 1994.

[9] M. J. Qin, M. W. D. Cooper, E. Y. Kuo, M. J. D. Rushton, R. W. Grimes, G. R. Lumpkin, and S. C. Middleburgh. Thermal conductivity and energetic recoils in $\mathrm{UO}_{2}$ using a many-body potential model. Journal of Physics: Condensed Matter, 26(49):495401, 2014.

[10] K. Une, S. Kashibe, and A. Takagi. Fission gas release behavior from high burnup $\mathrm{UO}_{2}$ fuels under rapid heating conditions. Journal of Nuclear Science and Technology, 43(9):1161$1171,2006$.

[11] J. A. Turnbull, S. K. Yagnik, M. Hirai, D. M. Staicu, and C. T. Walker. An Assessment of the Fuel Pulverization Threshold During LOCA-Type Temperature Transients. Nuclear Science and Engineering, 179(4):477-485, apr 2015.

[12] R.N. Singh and R. L. Coble. Growth of uranium dioxide single crystals by chemical vapor deposition. Journal of Crystal Growth, 21(2):261-266, feb 1974.

[13] T. L. Einstein. Equilibrium Shape of Crystals. In Handbook of Crystal Growth, pages 215264. Elsevier, second edition, 2015.

[14] T. R. Padden. An electron microscopy technique for studying shape, size and distribution of pores in sintered $\mathrm{UO}_{2}$ compacts. Technical report, WAPD-T-586. Westinghouse Electric Corp. Atomic Power Div., Pittsburgh, 1960.

[15] J. Belle and B. Lustman. Properties of $\mathrm{UO}_{2}$. Atomic Power Laboratory and U.S. Atomic Energy Commission, 1957.

[16] M. R. Castell. Wulff shape of microscopic voids in $\mathrm{UO}_{2}$ crystals. Physical Review B, 68(23):235411, dec 2003.

[17] K. T. Moore. X-ray and electron microscopy of actinide materials. Micron, 41(4):336-358, jun 2010.

[18] S. Plimpton. Fast Parallel Algorithms for Short-Range Molecular Dynamics, 1995.

[19] J. D Gale. GULP: A computer program for the symmetry-adapted simulation of solids. Journal of the Chemical Society, Faraday Transactions, 93(4):629-637, 1997.

[20] M. W. D. Cooper, M. J. D. Rushton, and R. W. Grimes. A many-body potential approach to modelling the thermomechanical properties of actinide oxides. Journal of Physics: Condensed Matter, 26(10):105401, mar 2014.

[21] M. W. D. Cooper, N. Kuganathan, P. A. Burr, M. J. D. Rushton, R. W. Grimes, C. R. Stanek, and D. A. Andersson. Development of Xe and $\mathrm{Kr}$ empirical potentials for $\mathrm{CeO}_{2}, \mathrm{ThO}_{2}$, $\mathrm{UO}_{2}$ and $\mathrm{PuO}_{2}$, combining DFT with high temperature MD. Journal of Physics: Condensed Matter, 28(40):405401, oct 2016.

[22] K. T. Tang and J. P. Toennies. The van der Waals potentials between all the rare gas atoms from He to Rn. Journal of Chemical Physics, 118(11):4976-4983, 2003.

[23] P. W. Tasker. The stability of ionic crystal surfaces. Journal of Physics C: Solid State Physics, 12(22):4977-4984, nov 1979. 
[24] M. Abramowski, R. W. Grimes, and S. L. Owens. Morphology of $\mathrm{UO}_{2}$. Journal of Nuclear Materials, 275(1):12-18, oct 1999.

[25] C. P. Robert and G. Casella. The Metropolis-Hastings Algorithm. Number Mcmc, pages 231-283. 1999.

[26] S. Marchio, S. Meloni, A. Giacomello, C. Valeriani, C. M. Casciola. Pressure control in interfacial systems: Atomistic simulations of vapor nucleation. Journal of Chemical Physics, 148(6):064706, feb 2018.

[27] J O Rourke. Computational Geometry in C, volume 53. Cambridge University Press, Cambridge, 1998.

[28] R. O. A. Hall, M. J. Mortimer, and D. A. Mortimer. Surface energy measurements on $\mathrm{UO}_{2}$ - A critical review. Journal of Nuclear Materials, 148(3):237-256, may 1987.

[29] W. Van Lierde. On the surface free energy anisotropy of UO2. Journal of Materials Science, 5(6):527-531, jun 1970.

[30] H. Serizawa, J. Matsunaga, Y. Haga, K. Nakajima, M. Akabori, T. Tsuru, Y. Kaji, S. Kashibe, Y. Ohisi, and S. Yamanaka. Formation and Growth of Image Crystals by Helium Precipitation. Crystal Growth \& Design, 13(7):2815-2823, jul 2013.

[31] R. G. Robins, B. S. Wilks, and B. T. Bradbury. The crystal habit of uranium dioxide. Journal of Nuclear Materials, 5(2):262-263, feb 1962.

[32] S. Takahashi. Micro-structures of Sintered Uranium Dioxide Pellets. US Atomic Energy Commission., 1961.

[33] A. S. Boyarchenkov, S. I. Potashnikov, K. A. Nekrasov, and A. Ya. Kupryazhkin. Molecular dynamics simulation of $\mathrm{UO}_{2}$ nanocrystals surface. Journal of Nuclear Materials, 421(1-3):18, feb 2012.

[34] A. Jelea, M. Colbert, F. Ribeiro, G. Tréglia, and R. J.-M. Pellenq. An atomistic modelling of the porosity impact on $\mathrm{UO}_{2}$ matrix macroscopic properties. Journal of Nuclear Materials, 415(2):210-216, aug 2011.

[35] G. Sattonnay and R. Tétot. Bulk, surface and point defect properties in $\mathrm{UO}_{2}$ from a tightbinding variable-charge model. Journal of Physics: Condensed Matter, 25(12):125403, mar 2013.

[36] F. N. Skomurski, R. C. Ewing, A. L. Rohl, J. D. Gale, and U. Becker. Quantum mechanical vs. empirical potential modeling of uranium dioxide $\left(\mathrm{UO}_{2}\right)$ surfaces: (111), (110), and (100). American Mineralogist, 91(11-12):1761-1772, nov 2006.

[37] A. H. H. Tan, M. Abramowski, R. W. Grimes, and S. Owens. Surface defect configurations on the (100) dipolar surface of $\mathrm{UO}_{2}$. Physical Review B, 72(3):035457, jul 2005.

[38] P. W. Tasker. The structure and properties of fluorite crystal surfaces. Le Journal de Physique Colloques, 41(C6):C6-488-C6-491, jul 1980.

[39] A. Chartier, L. Van Brutzel, and M. Freyss. Atomistic study of stability of xenon nanoclusters in uranium oxide. Physical Review B, 81(17):174111, may 2010. 
[40] A. Stukowski. Visualization and analysis of atomistic simulation data with OVITO-the Open Visualization Tool. Modelling and Simulation in Materials Science and Engineering, 18(1):015012, jan 2010.

[41] A. B. Belonoshko, S. Davis, A. Rosengren, R. Ahuja, B. Johansson, S. I. Simak, L. Burakovsky and D. L. Preston. Xenon melting: Density functional theory versus diamond anvil cell experiments. Physical Review B, 74(5):054114, aug 2006.

[42] J. Zheng, Q. F. Chen, Y. J. Gu, Z. Y. Chen and C. J. Li. Thermodynamics, compressibility, and phase diagram: Shock compression of supercritical fluid xenon. Journal of Chemical Physics, 141(12):124201, sep 2014.

[43] Y. Zhu and H. Hallberg. Investigation of faceted void morphologies in $\mathrm{UO}_{2}$ by phase field modelling. Journal of Nuclear Materials, 467:113-120, dec 2015.

[44] Y. Miao, T. Yao, J. Lian, S. Zhu, S. Bhattacharya, A. Oaks, A. M. Yacout, and K. Mo. Nanocrystallization induced by high-energy heavy ion irradiation in $\mathrm{UO}_{2}$. Scripta Materialia, 155:169-174, oct 2018.

[45] L. He, X. M. Bai, J. Pakarinen, B. J. Jaques, J. Gan, A. T. Nelson, A. El-Azab, and T. R. Allen. Bubble evolution in $\mathrm{Kr}$-irradiated $\mathrm{UO}_{2}$ during annealing. Journal of Nuclear Materials, 496:242-250, dec 2017.

[46] C. Baker. The migration of intragranular fission gas bubbles in irradiated uranium dioxide. Journal of Nuclear Materials, 71(1):117-123, dec 1977.

[47] M. H. Wood. An analysis of observations of fission gas bubble distributions in fuel. Journal of Nuclear Materials, 82(2):257-263, jul 1979.

[48] P. J. Goodhew. The shape of an overpressurized bubble. Journal of Nuclear Materials, 98(12):221-222, may 1981 .

[49] L. Van Brutzel and A. Chartier. A new equation of state for helium nanobubbles embedded in $\mathrm{UO}_{2}$ matrix calculated via molecular dynamics simulations. Journal of Nuclear Materials, 518:431-439, may 2019. 